Témoigner Témoigner. Entre histoire et mémoire

Getuigen Revue pluridisciplinaire de la Fondation Auschwitz

$122 \mid 2016$

Révisionisme et négationisme

\title{
Post. Une pièce de Solange Nebenzahl
}

Post

\section{Daniel Weyssow}

\section{(2) OpenEdition}

\section{Journals}

Édition électronique

URL : https://journals.openedition.org/temoigner/4015

DOI : 10.4000/temoigner.4015

ISSN : 2506-6390

Éditeur :

Éditions du Centre d'études et de documentation Mémoire d'Auschwitz, Éditions Kimé

Édition imprimée

Date de publication : 2 mai 2016

Pagination : 26-29

ISSN : 2031-4183

Référence électronique

Daniel Weyssow, «Post. Une pièce de Solange Nebenzahl », Témoigner. Entre histoire et mémoire [En ligne], 122 | 2016, mis en ligne le 30 septembre 2021, consulté le 03 janvier 2022. URL : http:// journals.openedition.org/temoigner/4015; DOI : https://doi.org/10.4000/temoigner.4015 


\section{POST}

THEATRE La pièce décrit, au fil de lettres rédigées par des dénonciateurs et par leurs victimes, lues par les comédiens, le processus de déportation des Juifs mis en place par l'occupant à Bruxelles au temps de la Seconde Guerre mondiale.

$\rightarrow$ Une pièce de Solange Nebenzahl

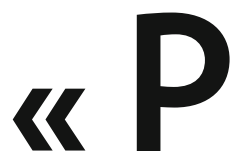

ost». Pourquoi ce titre? Tout d'abord, bien sûr, en référence aux services postaux. Entre expéditeur et destinataire, le rôle d'intermédiaire de la poste pour la distribution du courrier est bien entendu central. L'intitulé, ensuite, renvoie, dans la pièce, aux colis et lettres envoyés par les familles aux personnes séquestrées à la caserne Dossin et aux réponses de ces dernières. Enfin, le terme «post» fait appel à l' «après », c'est-à-dire à notre temps. Que nous disent, que nous apportent aujourd'hui ces lettres? Comment les rendre parlantes, utiles à nos contemporains?

Peut-on imaginer les activités souterraines qui avaient lieu dans les bureaux de tri de la poste centrale? Au cœur de l'appareil de distribution du courrier se trouvaient, bien heureusement, des membres de la Résistance, dont le rôle était d'intercepter et d'ouvrir les lettres de collaborateurs envoyées aux polices de l'occupant, Feldgendarmerie, Geheimfeldpolizei et Gestapo. «Des renseignements qui nous parvenaient, dévoilant des dénonciations, des lettres détournées par les postiers et des moyens encore plus compliqués nous permettaient d'agir et de protéger notre travail.» ${ }^{1}$

Le décor de la pièce semble à première vue sommaire, pourtant il n'en est rien. Des lettres, par centaines, tapissent les trois murs qui délimitent le plateau de scène. Quatre comédiens s'y trouvent, debout, côte à côte. Entre eux, en retrait, une musicienne, violoniste. La pièce débute par la projection vidéo d'un témoignage. Une femme âgée fait le récit de sa vie.

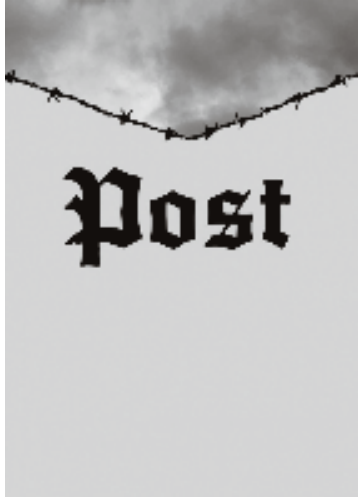

_-Affiche du spectacle

Ses parents, originaires de Slovaquie, sont arrivés en 1923 à Anvers, alors qu'ils n’avaient pas 20 ans. Elle et sa sœur sont nées dans les années trente. Elle décrit son quotidien jusquau déménagement de la famille rue Jakob Jordaens, où arriva cette terrible nuit de 1942 où les $\mathrm{SS}$ vinrent les rafler.

Sur scène, la projection s'estompe alors, et c'est un disque, rayé et grésillant, qui prend le relais, laissant place à un son d'époque. L'aiguille rebondit, rejouant à chaque retour de la griffe marquée dans le disque, ad aeternam, le même refrain. La vie hoquette, sans plus vraiment de respiration, presque à l'arrêt. Le temps se vide de toute substance, la mort rôde. Le violon électrique de la musicienne s'éveille alors, relayant en les amplifiant les circonvolutions sonores hypnotiques. Répétitives, elles semblent explorer de nouveaux espaces aussi tragiques qu'infinis.

À l'auteur d'une lettre de dénonciation reçue, exceptionnellement signée, l'on entend un des comédiens lire la réponse que lui adresse «monsieur» Hess, commissaire aux questions juives :

Madame,j'accuse réception de votre lettre du 22 courant. Elle a retenu toute mon attention. Quoique les textes qui sont en vigueur actuellement ne me permettent pas

(1) «Les Enfants », rapport écrit en novembre 1944 par Maurice Heiber, responsable du CDJ (Comité de Défense des Juifs), in Résistance. Père Bruno Reynders. Juste des Nations, Bruxelles, Les Carrefours de La Cité, juin 1993, p. 122. 
d'obliger un Juif à faire la preuve de sa religion, je tiens à vous faire savoir que cette question ne nous échappe pas et qu'un projet de loi devrait être établi sous peu. En ce qui concerne votre problème, j’ordonne qu'on mène l'enquête, et je vous promets de demander l'expulsion de Craenen s'il est juif. Soyez assuré que mon commissariat, malgré toutes les difficultés qu'il rencontre, résoudra sous peu et de manière radicale le problème juif en Belgique. Veuillez agréer Madame, mes salutations distinguées.

D’autres lettres, toujours plus nombreuses, affluent et défilent.

\begin{abstract}
À la Feldgendarmerie de Nivelles. Messieurs. Vous cherchez monsieur Stern à Braine-le-château ? Je vous fais savoir qu'il travaille à Wauthier-Braine. Il vient dans sa maison mais n’y loge pas. Il se trouve à la rue des brûlés, 16. C'est là que son épouse porte sa nourriture. Sincères salutations.
\end{abstract}

Le processus de déportation enclenché, après la rafle, c'est l'enfermement à la Caserne Dossin, puis le convoi vers Auschwitz. C'est que le parcours des victimes suit un cheminement bien déterminé...

Le dispositif scénique de Daniela Biscontipermet au public de prendre conscience des échanges épistolaires transitant par la poste centrale. Punaisées aux murs de la scène, les lettres tapissent en fait une structure de bois croisés. Les comédiens arrachent une par une les missives des parois pour les lire à tour de rôle. Leurs contenus transmis au public, elles ne sont pas replacées au mur. La transmission s'effectue, dans le cadre de la pièce, vers les spectateurs. Cette mémoire, à présent, appartient à qui l'a reçue. En tant que nouveaux dépositaires, comment allons-nous nous y prendre pour que cette connaissance puisse fructifier, pour que l'humanité sache, mieux armée, croître, si possible, en vertu et en sagesse? Les lettres lues sont évacuées. Jetées à terre ou plus loin, au travers des trous désormais apparents ouverts dans les parois, là où les lettres étaient fixées. Les murs, ainsi, s'allègent tandis que l'atmosphère s'alourdit du poids des mots qui envahissent la salle. Chaque lettre laisse ainsi place à un trou signalant une absence, un vide, une disparition. Les personnes dénoncées, envoyées à une mort quasi certaine dans les camps nazis, sont rappelées de la sorte, symboliquement, par un procédé qui a déjà une longue histoire. En 1969 déjà, George Perec, dans son roman intitulé La Disparition, avait évoquél' «absence» des Juifs dans le corps même de son récit en le rédigeant sans jamais utiliser la lettre «e». Au cinéma, Claude Lanzmann dans Shoah (1985), tout comme aujourd'hui Lászlo Nemes dans Le Fils de Saul (2015), ont cherché à faire apparaître la disparition en la circonscrivant. Appel est fait à l'imagination du spectateur pour qu'il «ressente» ou visualise l'espace de l'absence, le vide vacant. Des monuments ont également été conçus sur ce mode, à l'exemple de la Maison manquante de Christian Boltanski (Berlin, 1990) ou du Monument contre le racisme. 2146 pierres de Jochen Gerz (Saarebruck, 1993). Ces pavés sont disposés sur une place publique. Chacun porte le nom gravé d'un cimetière juif détruit - effacé - par les Allemands. Les inscriptions disposées sur les faces enterrées des pavés sont donc invisibles.

Mais que nous racontent encore ces innombrables lettres qui nous traversent, à force, l'échine de part en part? Par exemple ceci :

Messieurs, malgré toute votre vigilance, vous ne voyez quand même pas très clair. Tous les employés de la société Texaco sont fortement payés par la direction américaine. Il y a des Juifs parmi eux. Vous cherchez de la maind'œuvre bon marché pour l'Allemagne, là vous pouvez la trouver. Et en même temps vous mettrez la main sur des gens qui complotent certainement contre le Führer. Bonne chance. À vous. Et débarrassez-nous de ces poux.

La 8 erdonnance allemande du 29 mai 1942 impose aux Juifs de porter l'étoile jaune du côté gauche de la poitrine, et bien visiblement avec à l'intérieur l'inscription «Juif». Pourquoi mon voisin Ringer ne la porte pas? On ne va pas me dire qu'il ne l'a pas lue. Rue Haute, $n^{\circ} 6$, deuxième étage à droite. Vous savez où le trouver. À bon entendeur, salut.

Le dénommé Spitz, 14 rue de Mérode, porte l'étoile selon l'heure du jour, le soir pas, ce qui lui permet de faire ce qui lui plaît. Son fils vient parfois lui rendre visite sans porter l'étoile évidemment. À quand un coup de balai dans ce quartier infesté?

Monsieur, le Juif qui tient commerce à côté du mien me fait concurrence. Il vend à des clients des miroirs usagés qu'il déclare être neufs. A-t-il le droit de m’empêcher à moi, bon Belge, de gagner ma vie? Je veux croire que vous ferez le nécessaire pour l’en empêcher. Rue Blaes, 23. 
—. L'on ne peut que rester sidéré devant tant de méchanceté et de violence. Est-ce la jalousie ou l'appât du gain qui motivent de tels propos? De quel ordre est cette négation de l'humanité qui permet de traiter les Juifs de poux? Ces personnes n'avaientelles aucune conscience des conséquences de leurs actes? Ne savaient-elles pas qu'elles condamnaient à mort les personnes qu'elles désignaient à la vindicte policière de l'occupant? Bien sûr qu'elles savaient. On allait même, grâce à eux, chasser ces Juifs de leurs appartements... Ce que les dénonciateurs souhaitaient, c'était de les voir disparaître, pour empocher la prime promise, voire leurs biens. Ce qui arriverait aux personnes dénoncées n'avait pas la moindre importance à leurs yeux.

Ces lettres ont-elles été publiées ? Des recueils existent-ils ? Certaines proviennent du livre d'André Halimi, La délation sous l'occupation, d'autres, lues en néerlandais durant le spectacle, sont issues de l'ouvrage de Lieven Saerens, Steden in oorlog. Antwerpen 19401945. D'autres encore proviennent des archives du Centre Guerre et Sociétés contemporaines (Ceges), de la Caserne Dossin, du Musée Juif de Bruxelles, des archives de Maxime Steinberg et de celles de l'auteur de la pièce, Solange Nebenzahl. Elles sont ici bien entendu, dans le cadre de la pièce, remodelées et synthétisées.

Évoquer les dénonciations, et donc incidemment la collaboration, est une entreprise relativement récente au théâtre. Il n'est dès lors pas inutile de rappeler ici, en raison de la proximité des sujets traités, la pièce de Josse de Pauw, Ruhe («calme», en allemand) créée en 2007. Les chanteurs du Collegium vocale, interprétant des Lieder de Schubert, étaient répartis dans le public disposé en cercle autour d'un espace central dans lequel, soudainement, un acteur prit place pour y prononcer un monologue élaboréà partir de lettres de collaborateurs. Elles relatent, à l'aide d'arguments et de comparaisons aux « autres », les démocrates, pourquoi leurs auteurs préféraient et défendaient le nationalsocialisme, pourquoi ils collaboraient avec l'occupant et s'étaient engagés dans la SS. Leurs choix et leurs actions relevaient pour eux d'une évidence. Ils croyaient au futur d'une société national-socialiste, à la puissance et à la supériorité d'une communauté « aryenne » dont on leur disait qu'ils faisaient partie. Ils voulaient en finir avec les Juifs, le communisme et l'internationalisme, et voulaient être associés à une Europe «allemande». Évidemment, les justifications entendues passaient par la critique d'une société démocratique considérée comme trop faible à leurs yeux. Un terrible constat.

Aux côtés des lettres de dénonciation s'en trouvaient donc d'autres rédigées par les victimes, raflées, des dénonciateurs. Prises au piège, angoissées, manquant de tout, leur sort ne leur apparaissait peut-être pas très clairement. Elles ne s'attendaient peut-être pas au pire à l'époque des premiers convois, à voir leurs lettres, pour la plupart teintées d'espoir. Expédiées de la Caserne Dossin à Malines à destination de parents, elles véhiculaient des demandes de vivre et de vêtements, des remerciements pour les colis reçus, ou plus simplement des nouvelles ${ }^{3}$ :

Maman pourquoi les Allemands ont-ils des chiens qui font peur? J'ai peur des chiens. Tu crois qu'ils peuvent mordre? Ils m'ont pris à l'arrivée ma petite chaîne, celle que tu m'avais offerte pour ma Bar Mitsvah. Tu parles d'un accueil. «Aufnahme» comme ils disent. Pourquoi ils prennent nos choses? Dis maman tu m'en donneras une autre? Quand est-ce que tu viendras nous voir? C’est loin

(2) «Remercie toutes celles de la classe qui ont contribué pour les colis. Ma petite Maggy, je ne voudrais pas te donner l'impression que je suis découragée, mais je t'avoue que je traverse de terribles moments. L'avenir est encore si sombre, malgré tout. Je voudrais que tu me voies ici à l'écrire, cela ne doit pas être très fameux. Je termine. J'écrirai encore une carte au cas où celle-ci ne te parviendrait pas. Embrasse toute ta chère famille, les miens et pour toi, ma très chère, je te serre dans mes bras de toutes mes forces. Au revoir. Ita. »

"Train passé à Diest le 20.9 à 7h30. "

Extrait du livre de Simon Gronowski, L'enfant du $20^{\circ}$ convoi, Luc Pire, Bruxelles, 2002, p. 134.

(3) Les colis devaient être déposés par les familles à l'Association des Juifs en Belgique. Le personnel les faisait parvenir aux destinataires à la Caserne Dossin. 
la campagne? J'ai envie de rentrer à la maison. J'aimerais retourner à l'école. Papa me dit de te répéter de ne pas t'inquiéter. Envoie-moi un gâteau au fromage. Parfois je ferme les yeux et je vois ton sourire maman. S'il te plaît, viens nous chercher je ne veux pas partir, je veux rentrer à la maison. Je t’aime, Sara.

Je prie Madame D., directrice d'école, de me faire parvenir les articles suivant : une valise ou un panier, passemontagne chaud, bas sport chaud, longue culotte chaude, essuie-main, mouchoirs, moufles chaudes, savon, dentifrice, gourde, produits pharmaceutiques, si possible, urgent. Départ imminent. Conserves, vivres non périssables. De tout cœur merci.

Après le «séjour » à la caserne Dossin arrivait le moment d'embarquer dans un des convois vers Auschwitz. Des missives jetées des trains ont été ramassées et adressées aux destinataires :

Louise, Nous sommes dans un wagon, destination inconnue. Bon courage, prends soin de la petite.

Dites à tous et à toutes de ne pas se présenter à la caserne à Malines. Fuyez tant que vous le pouvez, Ferdinand.

D’autres lettres sont lues, rédigées par des condamnés à mort la veille de leur exécution :

Mes dernières heures ce 14 décembre. Maman que j’aimais tant, mon papa. Mes deux petites sœurs, vous qui ne me verrez plus, à quelques heures de mon exécution ma main ne tremble pas. J'attends. Dans quelques heures je serai une nouvelle et innocente victime. J'ai eu mes 21 ans loin de vous. J'ai réfléchi souvent aux peines que je vous ai données quand j'étais petit et quand vous guidiez mes premiers pas. Vous avez fait de moi j'ose l'écrire presque un homme. Et bien maman chérie c'est à toi que je veux écrire, il faut que tu vives, tu es nécessaire à mes deux petites sœurs, tu dois rester. Tu as été une mère exceptionnelle. Je crois plus que jamais à ceux que j’aime. Vivez, soyez heureux tous les 4, je le veux. Votre fils. Conservez mes affaires qui sont à la Kommandantur et à la Croix rouge. Courage, confiance.

Toutes ces lettres délivrent des messages visant soit à prévenir soit à rassurer les proches, à minimiser le malheur, ou à le repousser, sans doute pour ne pas trop y croire. En un mot, pour se donner du courage. Il est en

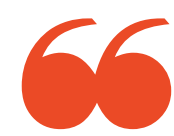

\section{Cher ami qui trouvera cette lettre. Pourrais-je te demander de la poster, même sans timbre?}

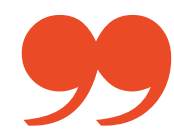

tout cas possible, à les entendre, de se rendre compte de l'état d'esprit des victimes, alors qu'on ne pourrajamais saisir le fond de la pensée des dénonciateurs et de leurs gestes assassins.

La pièce apporte beaucoup d'un point de vue pédagogique dans la mesure où elle permet d'aborder les traitements qu'ont subis les Juifs et les Résistants durant la Seconde Guerre mondiale. Elle constitue un excellent point d'entrée pour aborder l'histoire de la Shoah en Belgique. On peut donc la conseiller à un public scolaire, ainsi qu’à tous ceux qui peuvent entendre la litanie des drames que résument ces lettres dispensées à chaque étape de la chaîne du crime organisée par les nazis et leurs collaborateurs, mais aussi les cris d’appel au courage des populations juives décimées et des résistants que nul jamais ne devrait oublier.

«Post» s'achève sur les images qui concluent l'enregistrement vidéo du témoin présenté en introduction de la pièce. Son message porte sur la notion d'optimisme, une attitude à cultiver avant toute autre considération si l'on veut survivre aux aléas de ce monde.

Daniel Weyssow

\section{Plus d'infos}

Une pièce de Solange Nebenzahl, mise en scène par Daniela Bisconti, interprétée par Catherine Claeys, Janine Godinas, Catherine Graindorge, Alexandre von Sivers, et Benjamin Vanslembroeck.

$\rightarrow$ Studio, Bozar, Rue Ravenstein 23, 1000 Bruxelles (7-9 septembre 2015 - vue le 9 septembre 2015). 